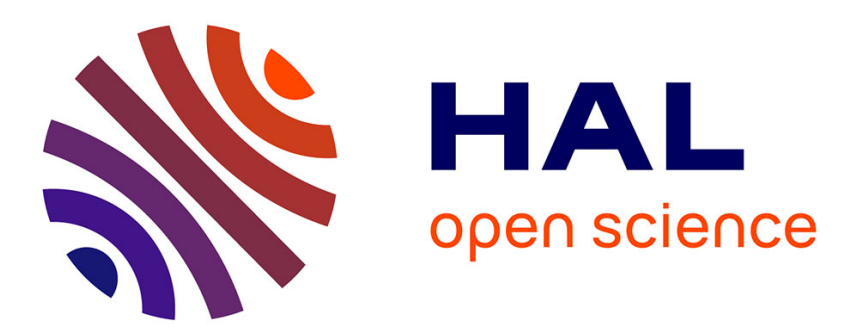

\title{
A limited number of 5-azacitidine cycles can be effective treatment in MDS
}

\author{
Catharina Müller-Thomas, Tibor Schuster, Christian Peschel, Katharina S.
}

Götze

\section{> To cite this version:}

Catharina Müller-Thomas, Tibor Schuster, Christian Peschel, Katharina S. Götze. A limited number of 5-azacitidine cycles can be effective treatment in MDS. Annals of Hematology, 2008, 88 (3), pp.213219. 10.1007/s00277-008-0583-8. hal-00477974

\section{HAL Id: hal-00477974 https://hal.science/hal-00477974}

Submitted on 30 Apr 2010

HAL is a multi-disciplinary open access archive for the deposit and dissemination of scientific research documents, whether they are published or not. The documents may come from teaching and research institutions in France or abroad, or from public or private research centers.
L'archive ouverte pluridisciplinaire HAL, est destinée au dépôt et à la diffusion de documents scientifiques de niveau recherche, publiés ou non, émanant des établissements d'enseignement et de recherche français ou étrangers, des laboratoires publics ou privés. 


\title{
A limited number of 5-azacitidine cycles can be effective treatment in MDS
}

\author{
Catharina Müller-Thomas • Tibor Schuster • \\ Christian Peschel • Katharina S. Götze
}

Received: 6 May 2008 / Accepted: 28 July 2008 / Published online: 12 August 2008

(C) Springer-Verlag 2008

\begin{abstract}
Hypomethylating agents, such as 5-azacitidine (5-AZA) and 5-aza-2'-deoxycytidine (decitabine), have recently been approved for the treatment of myelodysplastic syndromes (MDS). Several randomized trials have shown favorable results concerning response rate, survival, transformation to acute leukemia, and quality of life. In these trials, treatment was administered continuously until progression. In the retrospective study presented here, we evaluated the outcome of patients with higher risk MDS or secondary acute myeloid leukemia (sAML) treated with a limited number of 5-AZA cycles. A total of 32 patients received 5-AZA alone $(n=30)$ or in combination with valproic acid and all-trans retinoic acid $(n=2)$. 5-AZA was administered subcutaneously at a fixed dose of $75 \mathrm{mg} / \mathrm{m}^{2}$ daily for 7 days and repeated every 28 days. 5-AZA was given for a median of four courses. Treatment was continued for two more cycles as consolidation in patients who had achieved complete remission (CR), marrow $\mathrm{CR}$, or stable disease with hematologic improvement. The overall response rate was $50 \%$ according to the modified International Working Group criteria. Complete remissions were achieved in $15.6 \%$ and stable disease in $34.4 \%$ of patients. Peripheral blood counts normalized in $6.3 \%$ of patients while hematologic improve-
\end{abstract}

Contributions: C. M.-T. collected and analyzed data. T.S. performed statistical analyses. C.P. advised on analysis and interpretation of the data. K.S.G. designed the study and analyzed data. C. M.-T. and K.S. G. wrote the paper.

C. Müller-Thomas $\cdot$ C. Peschel $\cdot$ K. S. Götze $(\square)$

III Department of Medicine, Technical University of Munich, Ismaningerstr. 15 ,

81675 Munich, Germany

e-mail: k.goetze@1rz.tum.de

T. Schuster

Institute for Statistics, Technical University of Munich,

Munich, Germany ment was achieved in $25 \%$. The median time to AML in responding patients was 45 weeks, while AML occurred after a median of 14 weeks in non-responding patients $(P=.038)$. The median survival of all patients was 60 weeks; the median survival of responders was 74 weeks compared with 26 weeks in non-responders $(P=.047)$. In this retrospective analysis, 5-AZA was associated with a survival advantage in responding patients with higher risk MDS or SAML. These favorable results suggest that patients may benefit even from a limited number of courses of 5-AZA. A randomized controlled clinical trial is required to prospectively validate these findings.

Keywords Myelodysplastic syndrome - MDS · AML . 5 -azacitidine $\cdot$ Epigenetic therapy $\cdot$ Demethylation

\section{Introduction}

The myelodysplastic syndromes (MDS) encompass a heterogeneous group of clonal hematopoietic stem cell disorders associated with bone marrow failure, peripheral cytopenias, and a variable risk of progression into acute myeloid leukemia (AML). Patients suffer most commonly from anemia, followed by infectious complications due to neutropenia or bleeding because of thrombocytopenia. For many years, the main treatment option for MDS was best supportive care, which aims to alleviate the negative effects of cytopenia and to increase quality of life. Allogeneic hematopoietic stem cell transplantation is a curative treatment option but only available to a highly selected group of patients.

Recently, treatment modalities for MDS were expanded by the approval of the DNA methyltransferase inhibitors 5azacitidine (5-AZA) and 5-aza-2'-deoxyctidine (decitabine), 
which reverse abnormal DNA methylation [1]. Aberrant DNA methylation leads to gene silencing and loss of gene expression [2]. Silencing of tumor suppressor genes by hypermethylation has been shown to occur more frequently in cancer cells than normal cells [3, 4] and is thought to be an important mechanism in the development of MDS [5, 6]. Methyltransferase inhibitors are assumed to provide a selective tumor-specific mechanism for the restoration of normal gene function [7].

Several randomized trials with either 5-AZA or decitabine in patients with MDS have shown an overall response rate between $40 \%$ and $60 \%$, an improved quality of life, a reduced risk of transformation to AML, and a trend toward longer overall survival compared to best supportive care [8-14]. In these trials, treatment was continued indefinitely until disease progression occurred. Here, we report the results of a single center, retrospective data analysis of patients with higher risk MDS or secondary AML (sAML) treated with a limited number of 5-AZA cycles (or, in two cases, with 5-AZA, valproic acid and all-trans retinoic acid).

\section{Materials and methods}

Patients and therapy

Between August 2004 and December 2007, a total of 37 patients were treated with 5-AZA at our center. Clinical and hematologic data were obtained on 24 patients with MDS and eight patients with sAML. All patients had received no prior therapy for MDS or sAML except for best supportive care. MDS categories were determined according to the WHO classification. Patients with higher risk MDS (i.e., refractory anemia with excess blasts [RAEB] I and RAEB II as well as patients with poor risk cytogenetics according to WHO, with International Prognostic Scoring System [IPSS] Int-2 or higher) who were considered ineligible for more intensive chemotherapy were generally considered candidates for 5-AZA therapy.

Patients with lower risk MDS (i.e., refractory anemia [RA], refractory anemia with ringed sideroblasts [RARS], or refractory cytopenia with multilineage dysplasia [RCMD] with IPSS low or Int-1) were only treated if they had symptomatic anemia requiring regular red blood cell (RBC) transfusions or deterioration of preexisting thrombocytopenia or neutropenia to severe thrombocytopenia or neutropenia. Cytogenetics were available for 31 patients. Patients received 5-AZA subcutaneously at a fixed dose of $75 \mathrm{mg} / \mathrm{m}^{2}$ daily for 7 days. Valproic acid was given orally at $50 \mathrm{mg} / \mathrm{kg}$ daily for 7 days [15]. All-trans retinoic acid was given at $45 \mathrm{mg} / \mathrm{m}^{2}$ orally daily for 5 days, starting on day 3 [15]. Dexamethasone and granisetron were given as antiemetics $30 \mathrm{~min}$ before the administration of 5-AZA. Courses of therapy were repeated every 28 days.

A minimum of four courses was planned before response evaluation. Treatment was continued for two more cycles if a response was seen after four cycles (with the exception of one patient who received 13 cycles). No dose escalation was performed.

\section{Toxicity}

Hematologic toxicity was graded using relative changes in peripheral blood counts compared with those at treatment start (baseline): a decrease of $50 \%$ to $74 \%$ was defined as grade 3 and $75 \%$ and greater was defined as grade 4 [8]

\section{Response criteria}

Responses were evaluated according to the modified IWG response criteria [16]. The category "stable disease" was only used in patients with RAEB I, RAEB II, or sAML, while patients with low-risk MDS who did not show any hematologic improvement were classified in the category "no response". Treatment was considered to have failed in non-responders if they advanced to a higher WHO subtype or to sAML or died during treatment before bone marrow evaluation and without any hematologic improvement. Hematologic improvement was assessed according to the modified IWG response criteria [16].

\section{Statistical analysis}

Statistical analyses were done using SPSS Software (Version 15, SPSS Inc., Chicago, IL, USA). Survival rates were estimated by Kaplan-Meier method and median survival times were reported with $95 \%$ confidence intervals (CI). Log rank test was used for statistical comparisons of survival times between patient groups. To compare quantitative data between two independent samples, MannWhitney $U$ test was conducted. All statistical comparisons were performed two-sided. $P$ values less than 0.05 were considered statistically significant.

\section{Results}

Patient characteristics

Between August 2004 and December 2007, a total of 37 patients were treated with 5-AZA. The clinical and hematologic data of 32 patients were available for evaluation. Data on five patients were incomplete since these patients were lost to follow-up. Therefore, they could not be included in the analysis. Patient characteristics are shown in 
Table 1. Twenty-four (75\%) patients presented with MDS and eight $(25 \%)$ patients with secondary AML. IPSS risk and WHO classification-based Prognostic Scoring System (WPSS) risk were not categorized in ten patients $(31.3 \%)$ because eight of them had sAML, one of them had a dry marrow tap, and another one had inadequate bone marrow material to confirm the percentage of marrow blasts. The majority of patients treated constituted higher risk MDS as assessed by IPSS, WPSS, or WHO classification (Table 1). Sixteen $(50 \%)$ patients were transfusion dependent prior to therapy. The median age was 73.1 years and the male-tofemale ratio was $2: 1$.

\section{Treatment}

A total of 127 treatment courses were administered. The median number of courses was 4 (range 1-13 courses). Overall, 12 out of 32 patients $(37.5 \%)$ received less than four cycles of 5-AZA. Therapy was discontinued in ten of these 12 patients due to progression to overt leukemia or death. These patients were classified as treatment failures. The remaining two patients discontinued therapy before four cycles were completed due to side effects (patient choice). Of these two patients, one achieved stable disease and one had a marrow CR after two cycles. One patient received a total of 13 treatment courses. Thirty patients received 5-AZA and two patients were treated with 5AZA, valproic acid, and all-trans retinoic acid according to a recently published protocol [15]. Dose reduction to $75 \%$ was necessary in four patients due to prolonged thrombocytopenia.

\section{Clinical activity}

Responses are summarized in Table 2. An overall response rate of $50 \%$ was observed. This included two $(6.3 \%)$ complete remissions (CR), three (9.4\%) marrow CRs, and $11(34.4 \%)$ stable diseases (SD). Treatment failure was seen in $13(40.6 \%)$ patients. Three $(9.4 \%)$ patients with lower risk MDS (RA, RARS, and RCMD) did not have any hematologic improvement and were classified as nonresponders. Hematologic improvements were achieved in ten $(31.3 \%)$ patients. These included two (6.3\%) CRs, six $(18.8 \%)$ erythroid, platelet, or neutrophil responses, and two $(6.3 \%)$ relevant reductions in $\mathrm{RBC}$ transfusion requirement. When analyzing the response rate with respect to the IPSS (17), we obtained an overall response rate of $50 \%$ in the intermediate- 1 risk group $(n=6), 57.1 \%$ in the intermediate-2 risk group $(n=7)$, and $62.5 \%$ in the high-risk group $(n=8)$. The only patient in the low-risk group did not respond.

The recently established WPSS is a dynamic prognostic model based on WHO subgroups, karyotype and transfu-
Table 1 Patient characteristics

\begin{tabular}{|c|c|}
\hline Characteristics & Total $(\%)$ \\
\hline No. patients & 32 \\
\hline \multicolumn{2}{|l|}{ Age, year } \\
\hline Median & 73.1 \\
\hline Range & $60-84$ \\
\hline \multicolumn{2}{|l|}{ Sex, no. $(\%)$} \\
\hline Female & $10(31.3)$ \\
\hline Male & $22(68.7)$ \\
\hline \multicolumn{2}{|l|}{ MDS subtype, no. (\%) } \\
\hline RA & $1(3.1)$ \\
\hline RARS & $1(3.1)$ \\
\hline RCMD & $5(15.6)$ \\
\hline RAEB-1 & $2(6.3)$ \\
\hline RAEB-2 & $14(43.8)$ \\
\hline Not assessable & $1(3.1)$ \\
\hline sAML, no. (\%) & $8(25)$ \\
\hline \multicolumn{2}{|l|}{ Karyotype, no. (\%) } \\
\hline Good & $15(46.9)$ \\
\hline Intermediate & $3(9.4)$ \\
\hline Poor & $13(40.6)$ \\
\hline Not categorized & $1(3.1)$ \\
\hline \multicolumn{2}{|l|}{ IPSS, no. (\%) } \\
\hline Low & $1(3.1)$ \\
\hline Intermediate-1 & $6(18.8)$ \\
\hline Intermediate-2 & $7(21.9)$ \\
\hline High & $8(25)$ \\
\hline Not categorized & $10(31.3)$ \\
\hline \multicolumn{2}{|l|}{ WPSS, no (\%) } \\
\hline Very low & $0(0)$ \\
\hline Low & $3(9.4)$ \\
\hline Intermediate & $5(15.6)$ \\
\hline High & $8(25)$ \\
\hline Very high & $6(18.8)$ \\
\hline Not categorized & $10(31.3)$ \\
\hline \multicolumn{2}{|l|}{ Initial neutrophil count } \\
\hline Median $(\mathrm{g} / \mathrm{l}]$ & 3.39 \\
\hline$<1.8 \mathrm{~g} / 1$, no. $(\%)$ & $21(65.6)$ \\
\hline$>1.8 \mathrm{~g} / 1$, no. $(\%)$ & $11(34.4)$ \\
\hline \multicolumn{2}{|l|}{ Initial RBC } \\
\hline Median (g/dl) & 9.20 \\
\hline$<10$ g/dl, no. (\%) & $24(75)$ \\
\hline$>10 \mathrm{~g} / \mathrm{dl}$, no. $(\%)$ & $8(25)$ \\
\hline \multicolumn{2}{|l|}{ Initial platelet count } \\
\hline Median [G/1] & 82.41 \\
\hline$<100$ g/l, no. (\%) & $26(81.3)$ \\
\hline$>100 \mathrm{~g} / 1$, no. $(\%)$ & $6(18.7)$ \\
\hline $\mathrm{RBC}$ transfusion dependence & $16(50)$ \\
\hline Total no. of courses & 127 \\
\hline Median & 4 \\
\hline
\end{tabular}

$M D S$ myelodysplastic syndrome, $R A$ refractory anemia, $R A R S$ refractory anemia with ringed sideroblasts, $R C M D$ refractory cytopenia with multilineage dysplasia, $R A E B$ refractory anemia with excess blasts, $S A M L$ secondary acute myeloid leukemia, IPSS International Prognostic Scoring System; WPSS WHO classification-based Prognostic Scoring System, $R B C$ red blood cells 
Table 2 Response to treatment

\begin{tabular}{lc}
\hline Response according to modified IWG criteria & Total (\%) \\
\hline CR & $2(6.3)^{\mathrm{a}}$ \\
Marrow CR & $3(9.4)$ \\
SD & $11(34.4)$ \\
Failure & $13(40.6)$ \\
No response & $3(9.4)$ \\
\hline
\end{tabular}

$I W G$ International Working Group, $C R$ complete remission, $S D$ stable disease

${ }^{\mathrm{a}}$ One patient also had complete cytogenetic remission

sion requirement [18]. The overall response rate according to the WPSS was $33.3 \%, 60 \%, 50 \%$ and $66,7 \%$ in the low, intermediate-, high-, and very high risk group $(n=3,5,8$, $6)$, respectively. The results were not statistically significant. There was no patient in the very low risk group. Six of eight patients with sAML were treated with 5-AZA alone. An overall response rate of $25 \%$ was observed. This included one $\mathrm{CR}$ and one marrow $\mathrm{CR}$. The other two patients were treated with the combination of 5-AZA, valproic acid, and all-trans retinoic acid [15]. One patient had stable disease after four courses of therapy (conversion to RAEB-I, hematologic improvement with a significant increase of platelets), the other patient failed.

Response according to cytogenetic subgroup

Pre-treatment cytogenetic characteristics are shown in Table 3. Karyotype abnormalities were categorized according to IPSS. Fifteen of $32(46.9 \%)$ evaluable patients had good risk karyotypes, three (9.4\%) intermediate, and 13 (40.6\%) poor risk karyotypes; the karyotype of one patient was not assessable. The responses according to cytogenetic risk group are summarized in Table 4 . In the subgroup with favorable cytogenetics $(n=15)$, the response rate was

Table 3 Pretreatment cytogenetic characteristics

\begin{tabular}{lc}
\hline Risk group according to IPPS & Total (\%) \\
\hline Low & $15(46.9)$ \\
Normal & $12(37.5)$ \\
Isolated del(5q) & $1(3.1)$ \\
Isolated del(20q) & $2(6.3)$ \\
Intermediate & $3(9.4)$ \\
Trisomy 8 & $2(6.3)$ \\
Other than trisomy 8 & $1(3.1)$ \\
High & $13(40.6)$ \\
Abnormal chromosome 7 & $4(12.5)$ \\
$\quad$ isolated or +1 additional abnormality) & \\
Complex & $9(28.1)$ \\
Not categorized & $1(3.1)$ \\
\hline
\end{tabular}

IPSS International Prognostic Scoring System
Table 4 Response to treatment with respect to karyotype

\begin{tabular}{lll}
\hline $\begin{array}{l}\text { Pretreatment } \\
\text { cytogenetics according } \\
\text { to IPSS }\end{array}$ & $\begin{array}{l}\text { Response according to } \\
\text { modified IWG criteria }\end{array}$ & $\begin{array}{c}\text { Total (\% within } \\
\text { the subgroup) }\end{array}$ \\
\hline Good & CR & $1(6.7)$ \\
& Marrow CR & $1(6.7)$ \\
& SD & $5(33.3)$ \\
& Failure & $5(33.3)$ \\
Intermediate & No response & $3(20.0)$ \\
& CR & $0(0)$ \\
& Marrow CR & $0(0)$ \\
Poor & SD & $2(66.7)$ \\
& Failure & $1(33.3)$ \\
& CR (also CyCR) & $1(7.7)$ \\
& Marrow CR & $1(7.7)$ \\
Not categorized & SD & $4(30.8)$ \\
\hline
\end{tabular}

IPSS International Prognostic Scoring System, IWG International Working Group, $C R$ complete remission, $C y C R$ cytogenetic complete remission, $S D$ stable disease

$46.7 \%$. One patient with isolated $\operatorname{del}(5 q)$ did not respond. Two patients with isolated del(20q) achieved hematologic improvement with transfusion independence.

Among the three patients with intermediate prognostic karyotype, $66.7 \%$ achieved a response. Two patients were with trisomy 8 as an isolated abnormality. One patient died after two courses of 5-AZA without any improvement, the other patient had stable disease without hematologic improvement after four courses of therapy. In the subgroup with poor cytogenetics $(n=13)$, the response rate was $46.2 \%$. When analyzing the subgroup of patients $(n=6)$ with abnormal chromosome 7, we saw only one patient responding to therapy (marrow $\mathrm{CR}$ ). One patient with complex karyotype achieved a complete cytogenetic response. A total of seven (21.9\%) patients had complex abnormalities with involvement of $\operatorname{del}(5 q)$. In this subgroup, the response rate was $42.9 \%$.

Time to AML and overall survival

Ten patients developed secondary AML or relapsed from AML. For responding patients, the median time to AML progression was 45 weeks (95\% CI 22-68), while the median time to AML in non-responders was 14 weeks (95\% CI 4-22). This difference was significant $(P=0.038)$. The median survival of all patients was 60 weeks (95\% CI 11-108) (Fig. 1); regarding the responders the median survival was 74 weeks (95\% CI 46-102) compared with 26 weeks $(95 \%$ CI $22-30)$ in non-responders $(P=$ 0.047) (Fig. 2). The median duration of response was 64 weeks (95\% CI 56 - 77 weeks). 


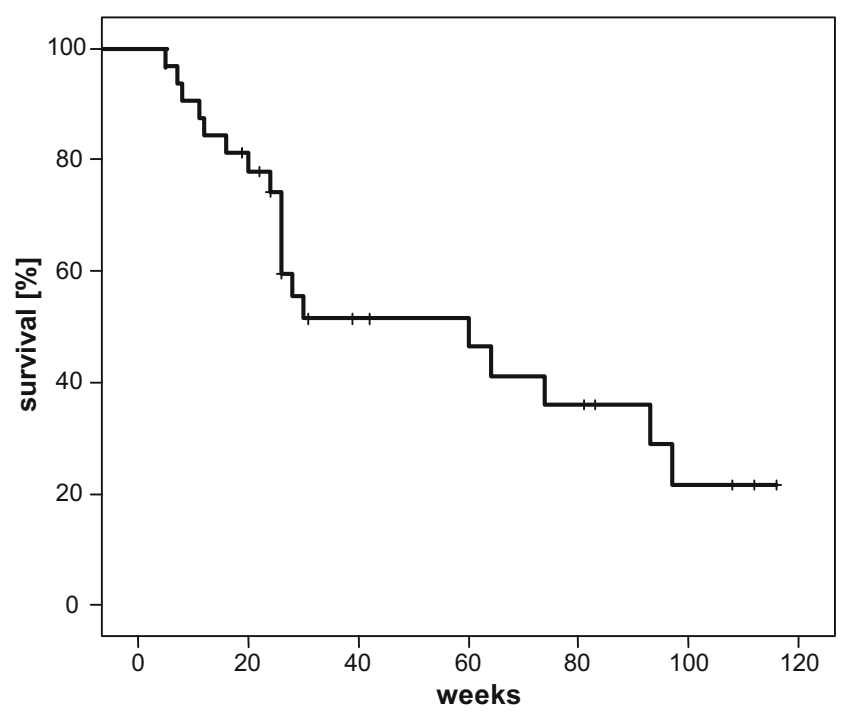

Fig. 1 Overall survival of all patients

\section{Toxicity}

The most common toxicity of 5-AZA is myelosuppression. In our patients, grade 3 or 4 neutropenia occurred in $21.9 \%$ and grade 3 or 4 thrombocytopenia in $40.6 \%$. Dose adjustment (reduction of $25 \%$ ) due to prolonged thrombocytopenia was necessary in four $(12.5 \%)$ patients. Subcutaneous administration of 5-AZA induced transient erythema at the injection sites in all patients. All patients received antiemetic prophylaxis with dexamethasone and granisetron so that most patients did not suffer from nausea or vomiting. Infectious complications during treatment with 5-AZA occurred in 14 (43.8\%) patients. From August 2004 to December 2007, 19 (59.4\%) deaths occurred. Three patients died during treatment (pneumonia, two; unknown, one). Ten deaths were attributed to secondary AML. Six deaths were due to infection $(n=1)$, bleeding $(n=1)$, and unknown reasons $(n=4)$.

\section{Discussion}

We conducted a retrospective data analysis of patients with MDS or SAML who were treated with 5-AZA. The median age of our patients was 73.1 years. Compared to patients included in the CALGB protocols 8421 and 9921, the median age in our patient cohort was higher by 8 and 4 years, respectively [19]. The higher median age in our patient group reflects the typical MDS patient population outside of a controlled clinical trial. The majority of patients had either higher risk MDS according to IPSS $(47 \%)$ or sAML $(25 \%)$.

The median number of courses administered was four. Treatment was continued for two more cycles as consoli- dation in patients who had achieved CR, marrow $\mathrm{CR}$, or SD with hematologic improvement. Since the first reported median time to response for 5-AZA was 3 cycles [19], treatment was stopped after four courses in patients with SD without hematologic improvement or at any time if failure was evident. Overall, 12 out of 32 patients (37.5\%) received less than four cycles of 5-AZA due to progression to overt leukemia or death. Only one patient received a total of 13 cycles due to continuous benefit, accounting for the range of 1-13 cycles.

The overall response rate was $50 \%$, consistent with published data $[8,19]$, with the majority of responding patients achieving stable disease. Compared to MDS studies 8421, 8921, and 9921 [19] the rate of complete remission (excluding marrow CR) in our analysis was clearly inferior $(6.3 \%$ vs $10-17 \%)$. However, we saw a high rate of stable disease (34.4\%). A likely explanation for these differences is the limited number of treatment courses in our center compared to the CALGB protocols. The recently published extended analysis of the MDS trials 8421,8921 , and 9921 revealed that the majority of responders (90\%) achieved a response by cycle 6 [19].

Although the median number of cycles administered in the Cancer and Leukemia Group B (CALGB) trials remains unclear (the reported range was 1-17 cycles), it can be assumed that it was higher than in our study since patients were treated until progression. The mechanism of action of 5 -azacitidine infers continuing benefit with prolonged therapy $[8,10,20]$. Nevertheless, even with the limited cycles of therapy administered to our patients, we observed a benefit in terms of time to AML progression and a statistically significant survival advantage for responders compared to non-responders (Fig. 2, 74 vs 26 weeks

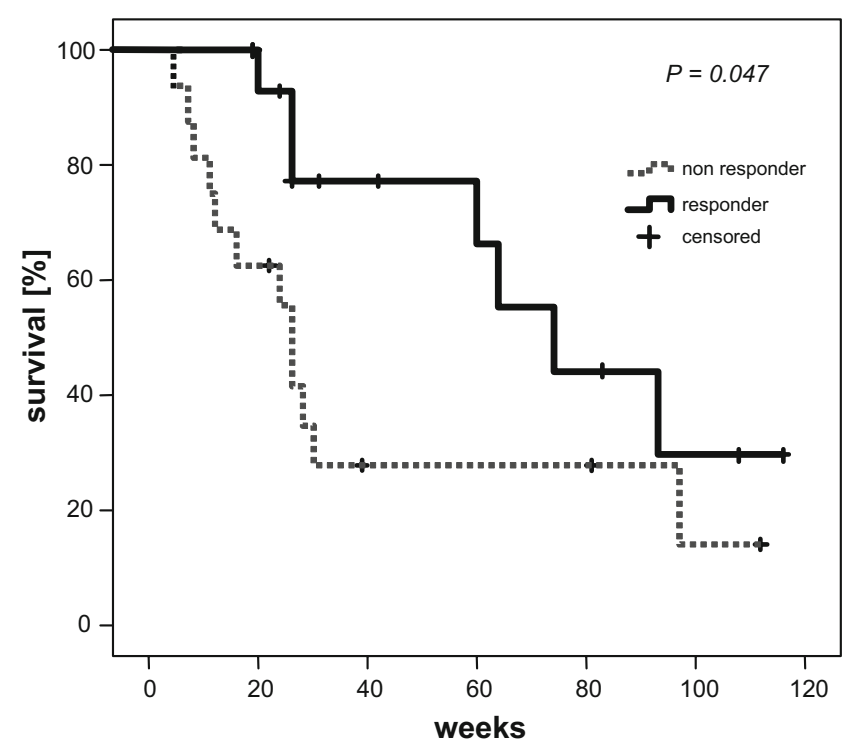

Fig. 2 Overall survival of responders and non-responders 
survival, respectively; $P=.047$ ) as well as a favorable response duration of 64 weeks. The observed median overall survival of 74 weeks in the group of responding patients corresponds well with the survival rates from the randomized phase II/III trials published for 5-AZA as well as decitabine [8, 10, 13, 14, 19], most of which had a higher number of treatment cycles. Therefore, our results suggest that even a limited number of treatment cycles may be beneficial for patients.

However, proof of the hypothesis that a defined shorter treatment is not inferior to treatment until progression requires confirmation by a randomized controlled clinical trial. This is especially true since the patients in our study were not treated within a defined protocol and therefore a selection bias cannot be completely excluded. The unfavorable median survival of non-responders seen in our study reflects the poor prognosis of patients with higher risk MDS [17].

The modified International Working Group (IWG) response criteria [16] provide a good basis for response evaluation. However, these might lack a category for lowrisk MDS not responding to treatment. "Stable disease" describes benefit from treatment in high-risk MDS as a temporary stop of disease progression, which may delay leukemic evolution. In low-risk MDS with a median overall survival of about 120 months "stable disease" cannot be seen as benefit from treatment. On the other hand, "failure" cannot be applied either as it assumes disease progression. For these reasons, we used the term "no response" for patients with low-risk MDS who did not benefit from treatment. Using this definition $60 \%$ of low-risk patients (RA, RARS, or RCMD with IPSS 0 or 1) showed no response.

Concerning the time to transformation to AML, a significant difference $(p=.038)$ was observed between responders (median, 45 weeks) and non-responders (median, 14 weeks). More responding $(n=6)$ than non-responding $(n=4)$ patients developed AML or relapsed from AML, which is explained by the fact that many non-responding patients died due to MDS-related causes (e.g., infections, bleeding) before leukemic evolution could occur.

No statistically significant differences were seen in response rate between the different risk groups with respect to IPSS, WPSS, and cytogenetic risk profile. We could also not confirm the superior response to demethylating therapy in patients carrying a chromosome 7 abnormality [21, 22]. However, the subgroups were probably too small to reach statistical significance.

In conclusion, 5-azacitidine was associated with a survival advantage in responding patients with MDS or sAML in this retrospective data analysis. Our results confirm the feasibility and beneficial effect of methyltransferase inhibitors on clinical outcome in a cohort of MDS patients substantially older than in the randomized CALGB trials.
Although we obtained a predominantly stable disease, we observed that patients benefited even from a limited number of treatment courses with 5-AZA. This may be explained by the fact that even disease stabilization confers an overall survival advantage for patients treated with 5-AZA, as shown by the recently presented data of the AZA-001 trial [23]. On the basis of these observations, continuous administration of 5-AZA until progression may not be necessary. We suggest that a randomized prospective controlled clinical trial be initiated to address this important question.

Acknowledgments We thank Ulrich Germing, Christian Meyer zum Büschenfelde, and Ulrich Keller for critical review of the manuscript and insightful discussions. We also thank Claudia Haferlach for cytogenetic analyses, and we are grateful to Peter Schmidkonz and Sigrun Gabius for providing clinical data and follow-up information. The authors have no conflict of interest and declare no competing financial interests.

\section{References}

1. Bhalla KN (2005) Epigenetic and chromatin modifiers as targeted therapy of hematologic malignancies. J Clin Oncol 23:3971-3993

2. Herman JG, Baylin SB (2003) Gene silencing in cancer in association with promoter hypermethlyation. N Engl J Med 349:2042-2054

3. Jones PA, Baylin SB (2002) The fundamental role of epigenetic events in cancer. Nat Rev 3(6):415-428

4. Antequera F, Boyes J, Bird A (1990) High levels of de novo methylation and altered chromatin structure at $\mathrm{CpG}$ islands in cell lines. Cell 62(3):503-514

5. Brakensiek K, Langer F, Schlegelberger B et al (2005) Hypermethylation of the suppressor of cytokine signalling-1 (SOCS-1) in myelodysplastic syndrome. Br J Haematol 130(2):209-217

6. Aoki E, Uchida T, Ohashi H, Nagai H, Murase T, Ichikawa A et al (2000) Methylation status of the p15INK4B gene in hematopoietic progenitors and peripheral blood cells in myelodysplastic syndromes. Leukemia 14(4):586-593

7. Cameron EE, Bachman KE, Myöhänen S, Herman JG, Baylin SB (1999) Synergy of demethylation and histone deacetylase inhibition in the re-expression of genes silenced in cancer. Nat Genet 21:103-107

8. Silverman LR, Demakos EP, Peterson BL, Kornblith AB, Holland JC, Odchimar-Reissig R et al (2002) Randomized controlled trial of azacitidine in patients with the myelodysplastic syndrome: a study of the cancer and leukemia group B. J Clin Oncol 20 (10):2429-2440

9. Wijermans P, Lubbert M, Verhoef G, Bosly A, Ravoet C, Andre $M$ et al (2000) Low-dose 5-aza-2' $\phi$-deoxycytidine, a DNA hypomethylating agent, for the treatment of high-risk myelodysplastic syndrome: a multicenter phase II study in elderly patients. J Clin Oncol 18(5):956-962

10. Kantarjian HM, Issa JP, Rosenfeld CS, Bennett JM, DiPersio MA, Klimek V et al (2006) Decitabine improves patient outcomes in myelodysplastic syndromes. Cancer 106(8):1794-1803

11. Kantarjian HM, Oki Y, Garcia-Manero G, Huang X, O'Brien S, Cortes J et al (2007) Results of a randomized study of 3 schedules of low-dose decitabine in higher-risk myelodysplastic syndrome and chronic myelomonocytic leukemia. Blood 109(1):52-57

12. Kornblith AB, Herndon JE 2nd, Silverman LR, Demakos EP, Odchimar-Reissig R, Holland JF et al (2002) Impact of azacyti- 
dine on the quality of life of patients with myelodysplastic syndrome treated in a randomized phase III trial: a Cancer and Leukemia Group B study. J Clin Oncol 20(10):2441-2452

13. Kantarjian HM, O'Brien S, Shan J, Aribi A, Garcia-Manero G, Jabbour E et al (2007) Update of the decitabine experience in higher risk myelodysplastic syndrome and analysis of prognostic factors associated with outcome. Cancer 109(2):265-273

14. Kantarjian HM, O’Brien S, Huang X, Garcia-Manero G, Ravandi F, Cortes J et al (2007) Survival advantage with decitabine versus intensive chemotherapy in patients with higher risk myelodysplastic syndrome. Cancer 109(6):1133-1137

15. Soriano AO, Yang H, Faderl S, Estrov Z, Giles F, Ravandi F et al (2007) Safety and clinical activity of the combination of 5azacytidine, valproic acid, and all-trans retinoic acid in acute myeloid leukemia and myelodysplastic syndrome. Blood 110 (7):2302-2308

16. Cheson BD, Greenberg PL, Bennett JM, Lowenberg B, Wijermans PW, Nimer SD et al (2006) Clinical application and proposal for modification of the International Working Group (IWG) response criteria in myelodysplasia. Blood 108(2):419-425

17. Greenberg P, Cox C, LeBeau MM, Fenaux P, Morel P, Sanz G et al (1997) International scoring system for evaluating prognosis in myelodysplastic syndromes. Blood 89(6):2079-2088
18. Malcovati L, Germing U, Kuendgen A, Della Porta MG, Pascutto C, Invernizzi R et al (2007) Time-dependent prognostic scoring system for predicting survival and leukemic evolution in myelodysplastic syndromes. J Clin Oncol 25(23):3503-3510

19. Silverman LR, McKenzie DR, Peterson BL, Holland JF, Backstrom JT, Beach CL et al (2006) Further analysis of trials with azacitidine in patients with myelodysplastic syndrome: studies 8421, 8921, and 9221 by the Cancer and Leukemia Group B. J Clin Oncol 24(24):3895-3903

20. Rueter B, Wijermans PW, Luebbert M (2006) Superiority of prolonged low-dose azanucleoside administration? Cancer 106(8):1744-1750

21. Rueter B, Wijermans P, Claus R, Kunzmann R, Luebbert M (2007) Preferential cytogenetic response to continuous intravenous low-dose decitabine (DAC) administration in myelodysplastic syndrome with monosomy 7. Blood 110(3):1080-1082

22. Raj K, John A, Ho A, Chronis C, Khan S, Samuel J, Pomplun S et al (2007) $C D K N 2 B$ methylation status and isolated chromosome 7 abnormalities predict responses to treatment with 5-azacytidine. Leukemia 21:1937-1944

23. List AF, Fenaux P, Mufti GF, Hellström-Lindberg E, Gore SD, Bennett JM et al (2008) Effect of azacitidine (AZA) on overall survival in higher-risk myelodysplastic syndromes (MDS) without complete remission. J Clin Oncol 26(15S), abstract 7006 